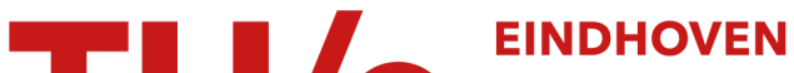

\section{Parity-check codes and post-processing for $d=1$ optical recording channels}

\section{Citation for published version (APA):}

Cai, K., Bergmans, J. W. M., Qin, Z., Padiy, A., \& Coene, W. M. J. (2005). Parity-check codes and postprocessing for $\mathrm{d}=1$ optical recording channels. IEEE Transactions on Magnetics, 41(11), 4338-4340. https://doi.org/10.1109/TMAG.2005.856587

DOI:

10.1109/TMAG.2005.856587

Document status and date:

Published: 01/01/2005

\section{Document Version:}

Publisher's PDF, also known as Version of Record (includes final page, issue and volume numbers)

\section{Please check the document version of this publication:}

- A submitted manuscript is the version of the article upon submission and before peer-review. There can be important differences between the submitted version and the official published version of record. People interested in the research are advised to contact the author for the final version of the publication, or visit the $\mathrm{DOI}$ to the publisher's website.

- The final author version and the galley proof are versions of the publication after peer review.

- The final published version features the final layout of the paper including the volume, issue and page numbers.

Link to publication

\section{General rights}

Copyright and moral rights for the publications made accessible in the public portal are retained by the authors and/or other copyright owners and it is a condition of accessing publications that users recognise and abide by the legal requirements associated with these rights.

- Users may download and print one copy of any publication from the public portal for the purpose of private study or research.

- You may not further distribute the material or use it for any profit-making activity or commercial gain

- You may freely distribute the URL identifying the publication in the public portal.

If the publication is distributed under the terms of Article 25fa of the Dutch Copyright Act, indicated by the "Taverne" license above, please follow below link for the End User Agreement:

www.tue.nl/taverne

Take down policy

If you believe that this document breaches copyright please contact us at:

openaccess@tue.nl

providing details and we will investigate your claim. 


\title{
Parity-Check Codes and Post-Processing for $d=1$ Optical Recording Channels
}

\author{
Kui Cai ${ }^{1}$, Jan W. M. Bergmans ${ }^{2}$, Zhiliang Qin ${ }^{1}$, Alexander Padiy ${ }^{3}$, and Wim Coene ${ }^{3}$ \\ ${ }^{1}$ Mechatronics and Recording Channel Division, Data Storage Institute, Singapore 117608 \\ ${ }^{2}$ Faculty of Electrical Engineering, Technische Universiteit Eindhoven, 5600 MB Eindhoven, The Netherlands \\ ${ }^{3}$ Storage Signal Processing Group, Philips Research Laboratories Eindhoven, NL-5656 AA Eindhoven, The Netherlands
}

We have developed an advanced detection approach with parity-check (PC) codes and post-processing for optical recording channels.
In seeking the advanced detection approach, we investigated different types of PC codes, existing as well as new. We developed a novel
remedy scheme to minimize the miscorrection of error events that are split across codeword boundaries. Simulation shows that the
performance of the developed PC codes approaches the corresponding bit-error-rate bounds, at both nominal density and high density.

Index Terms-Cyclic redundancy check (CRC) codes, matched-filtering type post-processor, parity-check codes.

\section{INTRODUCTION}

$\mathbf{I}^{\mathrm{N}}$ $\mathrm{N}$ recent years, the parity-check (PC) codes and post-Viterbi error correction processing based detection approach [1] has found wide acceptance in magnetic recording systems since it can correct dominant short error events at the channel detector output using only a few parity bits, and thereby significantly reduce the correction capacity loss of the error correction code (ECC). In this paper, we propose various PC codes in conjunction with improved post-processing for $d=1$ constrained optical recording systems (i.e., Blu-ray disc (BD) [2] or high-definition digital versatile disc (HD-DVD) [3]). In the study, we assume that the optical read-out is linear and use a generalized Braat-Hopkins model [4] to describe the channel. In the model, the quantity $\Omega_{u}=f_{c} T_{u}$, which is the optical cutoff frequency $f_{c}$ normalized by user bit rate $1 / T_{u}$, is a measure of the recording density. In this paper, cutoff frequencies $\Omega_{u}=$ 0.5 and $\Omega_{u}=0.375$ are considered. These choices represent recording systems with nominal density and high density, respectively, according to current standards. A Viterbi detector (VD) that is matched to a 7-tap optimized channel partial response (PR) target is used as the channel detector [5]. The additive channel noise before equalization is assumed to be Gaussian and white. The variance $\sigma_{n}^{2}$ of the channel noise is determined by the user signal-to-noise ratio $(\mathrm{SNR})$ defined as $\mathrm{SNR}_{\mathrm{u}}(\mathrm{dB})=$ $10 \log _{10}\left(\sum h_{k u}^{2}\right) /\left(\sigma_{u}^{2}\right)$ and $\sigma_{n}^{2}=(1 / R) \sigma_{u}^{2}$, where $\sigma_{u}^{2}$ is the noise power in the user bandwidth, $R$ is the code rate of the $d=1$ PC code, and $h_{k u}$ is the channel symbol response for $R=1$ and $\Omega_{u}=0.33$. The above definitions of SNR and channel response help to fairly reflect the impact of code rate in the system performance evaluation.

\section{PARITY-CHECK CODES}

\section{A. Cyclic Redundancy Check Codes}

Cyclic redundancy check (CRC) codes are effective and simple PC codes for error detection, and are defined by their generator polynomials $G(D)$. The suitability of different PC codes for a given channel and detector is determined by the error event distribution at the detector output. Using the theoretical

Digital Object Identifier 10.1109/TMAG.2005.856587
TABLE I

ERROR-DETECTION CAPABILITY OF VARIOUS CRC CODES

\begin{tabular}{|c|c|c|c|c|c|c|}
\hline \multirow{2}{*}{$\begin{array}{c}\text { Dominant } \\
\text { error events }\end{array}$} & \multicolumn{7}{|c|}{ CRC codes } \\
\cline { 2 - 7 } & $\mathrm{G}(D)=1+D$ & $\mathrm{G}(D)=1+D+D^{2}$ & $\mathrm{G}(D)=1+D+D^{3}$ & $\mathrm{G}(D)=1+D+D^{4}$ & $\mathrm{G}(D)=1+D^{7}$ & $\mathrm{G}(D)=1+D^{9}$ \\
\hline \hline$\{1,0,-1\}$ & $\times$ & $\sqrt{ }$ & $\sqrt{ }$ & $\sqrt{ }$ & $\sqrt{ }$ & $\sqrt{ }$ \\
\hline$\pm\{1\}$ & $\sqrt{ }$ & $\sqrt{ }$ & $\sqrt{ }$ & $\sqrt{ }$ & $\sqrt{ }$ & $\sqrt{ }$ \\
\hline$\pm\{1,0,-1,0,1\}$ & $\sqrt{ }$ & $\times$ & $\sqrt{ }$ & $\sqrt{ }$ & $\sqrt{ }$ & $\sqrt{ }$ \\
\hline$\pm\{1,0,-1,0,1,0,-1\}$ & $\times$ & $\sqrt{ }$ & $\sqrt{ }$ & $\sqrt{ }$ & $\sqrt{ }$ & $\sqrt{ }$ \\
\hline$\pm\{1,0,0,-1\}$ & $\times$ & $\times$ & $\sqrt{ }$ & $\sqrt{ }$ & $\sqrt{ }$ & $\sqrt{ }$ \\
\hline$\pm\{1,0,-1,0,1,0,-1,0,1\}$ & $\sqrt{ }$ & $\sqrt{ }$ & $\sqrt{ }$ & $\sqrt{ }$ & $\sqrt{ }$ & $\sqrt{ }$ \\
\hline
\end{tabular}

analysis described in [5], we can identify dominant error events at the VD output. Table I lists the first six dominant error events in nonreturn-to-zero (NRZ) format and with descending order of probabilities, for $\Omega_{u}=0.5$ and $\Omega_{u}=0.375$. Also listed are the capabilities of selected CRC codes in detecting these error events. In the table, a " $\sqrt{ }$ " denotes that the error event can be detected by the corresponding PC code, a " $X$ " denotes that the error event cannot be detected by the corresponding PC code. Observe that dominant error events in optical recording systems differ from those in magnetic recording. The event $\pm\{1,0,-1\}$ is the most dominant error event at high SNR or high recording density. By using three or more parity bits, we can detect all the error events listed in Table I.

When the parity-check constraint is imposed on constrained coded data sequences, an additional code rate loss will be incurred. For the rate $2 / 3 d=1$ codes [2], [3] used for BD and HD-DVD, the minimum overhead is 1.5 channel bits per parity bit. A good combination of PC codes with $d=1$ codes, such as the combi-code of [6], may achieve this minimum overhead. Therefore, we assume that each parity bit requires the minimum overhead of 1.5 channel bits. Let there be $r$ parity bits per codeword of length $N$. Then, the overall rate with parity-check coding is $R=(2 / 3)-(r / N)$. For the sake of simplicity, our study does not include an explicit constrained PC encoder. Instead, we model the PC codes by assuming that the parity bits for each codeword are known at the receiver. This is done by generating the syndrome for each codeword in a "data-aided" mode.

\section{B. New Parity-Check Codes}

Parity-check coding focuses on the dominant error events at the detector output. Clearly, the first dominant error event 
$\pm\{1,0,-1\}$ cannot be detected by the conventional single-bit even PC code with $G(D)=1+D$. Therefore, we propose a new single-bit PC code with parity-check matrix given by $\mathbf{H}=[1,1,0,0,1,1,0,0, \ldots, 1]$. It is easy to verify that this code can detect the event $\pm\{1,0,-1\}$ occurring at any position in the data sequence. Moreover, it can also detect the events $\pm\{1\}, \pm\{1,0,-1,0,1\}, \pm\{1,0,0,-1\}$, $\pm\{1,0,-1,0,1,0,-1,0,1\}$ listed in Table I, at specific positions of the data sequence. The proposed new single-bit PC code also facilitates the design of a two-level hierarchical parity-check coding scheme.

To enhance error detection and correction beyond the single-bit PC codes, multiple-bit PC codes need to be used. We model multiple-bit constrained PC codes by assuming a minimum rate loss of 1.5 channel bits per parity bit. However, for systems where such efficient constrained PC codes are difficult to construct, a hierarchical parity-check coding scheme may be used instead. In this paper, we propose a two-level hierarchical scheme. It arranges the data sequence into two data levels, according to the probabilities of the first dominant and the second dominant error events. We choose the new single-bit PC code, denoted by $P C 1$, to protect the first data level, since it can detect the event $\pm\{1,0,-1\}$. The single-bit even PC code, denoted by $P C 2$, is used to protect the second data level, since it can detect most of the remaining dominant error events. The overall code rate is given by

$$
R_{h}=\frac{2}{3}-\frac{r_{1}}{N_{1}}-\frac{r_{2}}{N_{2}}+\frac{r_{1} r_{2}}{N_{1} N_{2}}
$$

where $r_{i}$ and $N_{i}$ are the number of parity bits and data block length, respectively, for the $i$ th data levels, $i=1,2$. Usually, $N_{2} \gg r_{2}$ and $N_{1} N_{2} \gg r_{1} r_{2}$; hence, the code rate loss introduced by the parity check for the second data level is very small. Therefore, using the hierarchical scheme, more of the dominant error events can be detected, without incurring much rate loss. While decoding, we first carry out error correction with $P C 1$. Subsequently, we compute the syndrome and carry out error correction with $P C 2$. Note that the two component codes are all single-bit PC codes. Compared with the multiple-bit PC codes, they are easier to be combined effectively with the modulation constraints, since they have less stringent PC constraint (i.e., parity bits). Furthermore, the associated encoding/decoding complexity is also lower than that of the constrained multiple-bit PC codes. The two-level hierarchical scheme could be extended to multilevel schemes.

\section{Post-Processing ScheMes}

The optimum full state trellis detection [1] for the paritycheck coded channel is usually computational very expensive. Therefore, in this work, we use the simple maximum likelihood (ML) based matched-filtering type post-processor as the decoder of the PC code, which corrects errors by assuming that only one error event is present in a codeword [1]. Compared with magnetic recording systems with $d=0$ constraint, the optical recording system has a more stringent $d=1$ constraint. This constraint has been taken into consideration in both the VD and post-processor. In particular, for post-processing, in addition to the parity-check constraint, the outputs of the matched filters are also examined for the $d=1$ constraint. The maximum output that satisfies both constraints is used to locate the corresponding error event.

The impact of error events that are split across the boundary of two codewords is an issue that is of much concern for error correction. In this work, we investigate this issue and propose a remedy. The probability that error events occur at the boundary is given by

$$
P_{b_{-} \text {event }} \leq \sum_{\mathbf{e} \in \mathrm{E}} \frac{L_{e}-1}{N} U(\mathbf{e}) \operatorname{Pr}(\mathbf{e})
$$

where $\mathrm{E}$ is the set of error events at the detector output, and $U(\mathbf{e})$ is the probability of selecting an admissible data sequence a that supports a given error event e of length $L_{e}$ [5]. The codeword $\mathbf{a}$ is said to be admissible if $a_{i}=e_{i} / 2$, for $i=1,2, \ldots, N$, and if the erroneous word $\hat{\mathbf{a}}=\mathbf{a}-\mathbf{e}$ also satisfies the $d=1$ constraint. Here, $\operatorname{Pr}(\mathbf{e})$ is the probability that the detector input is closer to the input estimated using a than it is to the signal estimated using the correct sequence a [5]. The contribution of the boundary error events to the increase in bit-error rate (BER), compared to the performance of the ideal decoder [5], can be computed as

$$
P_{b_{-} \text {ber }} \leq \sum_{\mathbf{e} \in \mathrm{E}} \frac{L_{e}-1}{N} W(\mathbf{e}) U(\mathbf{e}) \operatorname{Pr}(\mathbf{e})
$$

where $W(\mathbf{e})$ is the number of nonzero elements in e. Computer simulations were carried out to verify the above analysis, and we found the theoretical calculations match the simulations very well.

To combat the impact of boundary error effect on the performance, a novel remedy scheme has been proposed. We divide each boundary error event into two parts: the leading part that lies at the end of the current data block, and the trailing part that lies in the beginning of the next data block. For example, if the error event length is 5, the leading part and the trailing part may last 4 bits at most. We note that the error patterns obtained by splitting the dominant error events in Table I are still in the list of error events given in this table. As described in Section II-A, by adding 3 or more parity bits, all these error events can be detected. Therefore, the parity check for each data block is still carried out from time index 1 to $N$. For error correction, we realized that if we simply treat the leading part and the trailing part as two separate error events, the erroneous bits in the trailing part may affect the correction of the leading part, and vice versa. Therefore, during error correction, we extend the current data block by a few bits more (e.g., 4 bits). This helps to determine the type and position of the boundary error event. Moreover, whenever we find that the detected error event extends across the block boundary, the $d=1$ constraint needs to be checked through the extended data block, while the parity-check constraint is checked within the original data block. Based on this, the erroneous bits in the leading part are corrected. For the trailing part of the boundary event, since the leading part has already been corrected, we treat it as a separate error event and correct it in the next data block.

\section{Simulation ReSults AND CONCLUSION}

For a given PC code, the choice of a suitable codeword length is a compromise between the code rate loss and error correction power. In this work, we chose to use a codeword length of $N_{0}=$ 
100 bits per parity bit. The corresponding code rate loss is $1.5 \%$. We find that such a selection is effective, since the probabilities of the dominant error events of the system are far smaller than $\left(1 / r N_{0}\right)$, and since the corresponding impact of rate loss on performance is found to be negligible. Along the same lines, we chose $N_{1}=100$ bits and $N_{2}=500$ bits for the proposed two-level hierarchical scheme.

From Fig. 1, we observe that compared with the performance of the system without PC code (Curve 1), the conventional single-bit even parity check code (Curve 2 ) provides no performance gain at all, since it cannot detect the dominant error event $\pm\{1,0,-1\}$. The proposed new single-bit code (Curve 3) and the two-level hierarchical scheme (Curve 4) provide about 0.25 and $0.75 \mathrm{~dB}$ gain in performance, respectively, at $\mathrm{BER}=10^{-5.5}$. The performance of the two-level hierarchical scheme is superior to that of its two components codes, and is similar to that of the PC code with $G(D)=1+D+D^{2}$. The reason is that the two codes have similar error detection capabilities, they provide similar amount of side information to the post-processor (with a syndrome of length 2), and meanwhile they have similar code rate loss. ${ }^{1}$ The PC codes with $G(D)=1+D+D^{4}$ (Curve 5) and $G(D)=1+D^{7}$ (Curve 6) achieve larger performance gains, since they can detect all the dominant error events listed in Table I and provide more side information to the post-processor. The longer syndromes provided by these codes help to decrease the miscorrection of the post-processor. The 7-bit code with $G(D)=1+D^{7}$ gains $1.2 \mathrm{~dB}$, while it lags $0.5 \mathrm{~dB}$ behind the performance bound (Curve 8). The bound corresponds to the case when all the dominant error events listed in Table I can be corrected, and is calculated analytically using [5]. The 9-bit code (Curve 7) does not provide significant performance gain over the 7-bit code. This is due to the occurrence of multiple error events per codeword of length $N=900$, which is beyond the correction capability of the post-processor. In addition, all the performances with PC codes are obtained using the proposed remedy scheme. It brings around $0.2 \mathrm{~dB}$ gain over the performances without remedy, which are not shown here for the sake of simplicity.

At $\Omega_{u}=0.375$, observe that compared with the results at nominal density, the performance gains are modest for most of the codes. On the other hand, the performance gaps of these codes from the corresponding bound are also reduced. In Fig. 2, the PC code with $G(D)=1+D^{7}$ achieves the best performance of $0.7 \mathrm{~dB}$ better than the case without PC code. It lags behind the bound by around $0.1 \mathrm{~dB}$. The rather modest performance gains observed at high density is because there are more nondominant error events at $\Omega_{u}=0.375$ than at $\Omega_{u}=0.5$. These nondominant error events are long error events with small probabilities; mostly are consecutive minimum distance transitions (i.e., $\pm\{1,1,-1,-1, \ldots\})$. To detect them, more parity bits are needed, or the underlying data patterns must be avoided by means of a suitable code constraint. They are also difficult to correct, since miscorrection of these long events will introduce many more errors.

\footnotetext{
${ }^{1}$ We do not show the performances of PC codes with $G(D)=1+D+D^{2}$ and $G(D)=1+D+D^{3}$, since they are found to be respectively very close to that of the two-level hierarchical scheme and the PC code with $G(D)=$ $1+D+D^{4}$.
}

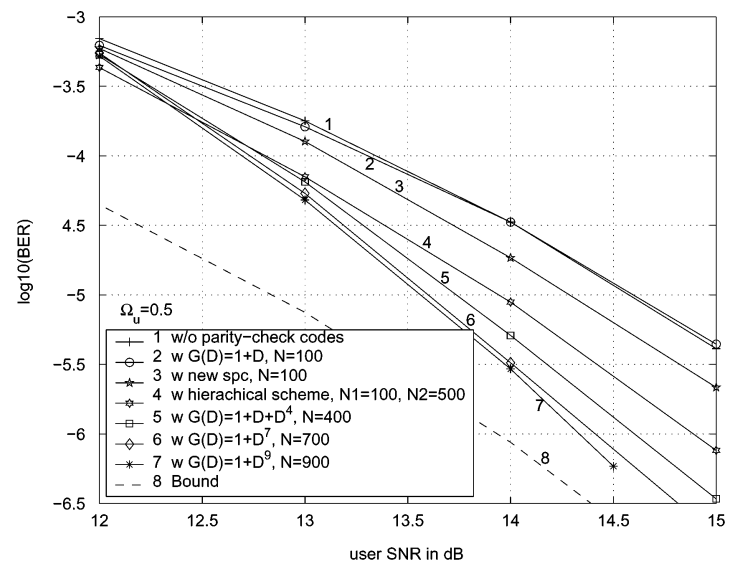

Fig. 1. BER performance of various PC codes, at nominal density.

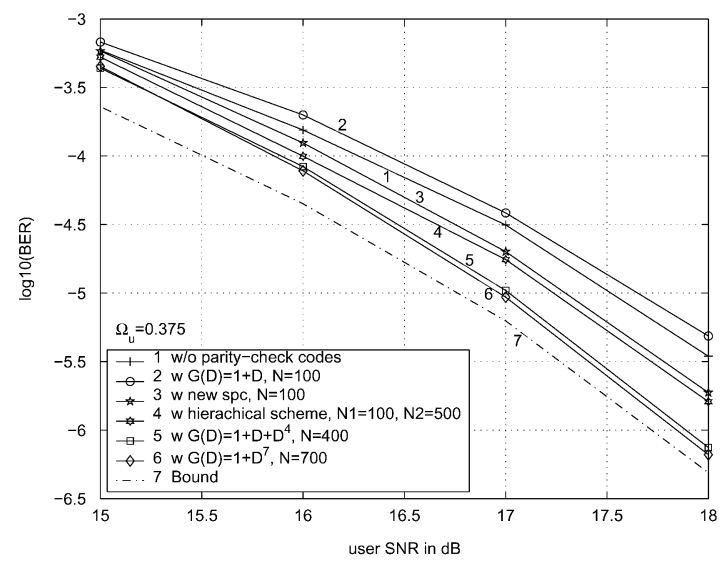

Fig. 2. BER performance of various PC codes, at high density.

\section{ACKNOWLEDGMENT}

This work was supported by the DSI-Philips SS Project.

\section{REFERENCES}

[1] R. D. Cideciyan, J. D. Coker, E. Eleftheriou, and R. L. Galbraith, "Noise predictive maximum likelihood detection combined with parity-based post-processing," IEEE Trans. Magn., vol. 37, no. 2, pp. 714-720, Mar. 2001.

[2] T. Narahara, S. Kobayashi, Y. Shimpuku, G. van den Enden, J. Kahlman, M. van Dijk, and R. van Woudenberg, "Optical disc system for digital video recording," Jpn. J. Appl. Phys., pt. 1, vol. 39, no. 2B, pp. 912-919, 2000.

[3] K. Kayanuma, C. Nota, and T. Iwanaga, "Eight to twelve modulation code for high density optical disk," in Tech. Dig. Int. Symp. Optical Memory. Nara, Japan, Nov. 2003, pp. 160-161.

[4] K. Cai, G. Mathew, J. W. M. Bergmans, and Z. Qin, "A generalized description of Braat-Hopkins model for optical recording channels," in Proc. IEEE Intl. Conf. Consumer Electronics. Los Angeles, CA, Jun. 2003, pp. 324-325.

[5] K. Cai, V. Y. Krachkovsky, and J. W. M. Bergmans, "Performance bounds for parity coded optical recording channels with $\mathrm{d}=1$ constraint," in Proc. IEEE Intl. Conf. Commun.. Alaska, May 2003, pp. 2914-2918.

[6] W. M. J. Coene, H. Pozidis, and J. W. M. Bergmans, "Run-length limited parity-check coding for transition-shift errors in optical recording," in Proc. IEEE Intl. Conf. Global Telecommun.. San Antonio, TX, Nov. 2001, pp. 2982-2986.

Manuscript received August 21, 2004; revised June 20, 2005. 